\title{
Prevalence of intravenous medication administration errors: a cross-sectional study
}

\author{
Tezeta Fekadu' \\ Mebrahtu Teweldemedhin ${ }^{2}$ \\ Eyerusalem Esrael' \\ Solomon Weldegebreal \\ Asgedom' \\ 'School of Pharmacy, Department \\ of Clinical Pharmacy, College of \\ Health Sciences, Mekelle University, \\ Mekelle, ${ }^{2}$ Unit of Biomedical Science, \\ School of Medicine, College of Health \\ Sciences and Referral Hospital, Aksum \\ University, Aksum, Ethiopia
}

Correspondence: Solomon Weldegebrea Asgedom

School of Pharmacy, Department of Clinical Pharmacy, College of Health Sciences, Mekelle University, Mekelle I87I, Ethiopia

Tel +25I 920871964

Email s.weldegebreal@gmail.com

\author{
This article was published in the following Dove Press journal: \\ Integrated Pharmacy Research and Practice \\ 31 January 2017 \\ Number of times this article has been viewed
}

\begin{abstract}
Background: Intravenous medication administration errors (MAEs) may be accompanied by avoidable undesirable effects, which might result in clinical complications.

Objective: The aim of the study was to determine the prevalence of MAEs and to identify the factors associated with such errors.

Methods: A hospital-based cross-sectional study was conducted from March to April 2015. Data were collected by direct observation using a pretested data collection tool. Simple random sampling was used, and bivariate logistic regression model was used to identify the factors associated with MAEs. $P$ value $<0.05$ was considered statistically significant.

Results: A total of 134 patients were found to be eligible for the study. More than half of the study participants were males (76 [56.7\%]). The rate of MAE was $46.1 \%$, with the missed dose $(n=162,95.8 \%)$ being reported as the most common error. The age groups of 60-79 years (adjusted odds ratio $=2.166$, confidence interval $=1.532-8.799$ ) and $80-101$ years (adjusted odds ratio $=1.52$, confidence interval $=1.198-5.584$ ) were the determinants of MAEs.

Conclusion: A high prevalence of MAEs was found. Enhancing the knowledge and practical skills of clinical nurses might minimize such errors.
\end{abstract}

Keywords: prevalence, medication, administration, error

\section{Background}

Preparation and intravenous (IV) administration of medications is a crucial clinical activity in provision of health care for hospitalized patients. ${ }^{1,2}$ In health care systems, the medication administration processes represent an advanced technology and a complex process. ${ }^{3,4}$ Infusion therapy is one of the medication administration processes used in the treatment of many hospitalized patients; however, it is associated with high risk of causing harm for patients. ${ }^{5,6}$ Administration of medications may be accompanied by avoidable undesirable effects which sometimes are life threatening, especially when administered in error. ${ }^{7,8}$

Medication errors are among the major clinical issues in the health care settings. ${ }^{9-11}$ Although IV therapy benefits patients, it also represents a source of risks. The complexity of the procedures, multiplicity of professionals and services involved, rapid introduction of new drugs, and diagnostic and therapeutic technologies frequently provoke errors, jeopardizing care security and quality and generating an increase in cost. ${ }^{3,12}$

Medication errors are the eighth leading cause of death in the US, and they represent the single largest cause of errors in the hospital setting, accounting for $>7000$ deaths annually. ${ }^{13,14}$ In the US, $60 \%$ of serious and life-threatening medication errors that occur in patients involved IV drugs; in the UK approximately $56 \%$ of the errors 
administered with IV drugs. Although only a few medications are administered IV in the hospital setting, the IV drugs account for the majority of medication errors. ${ }^{15,16} \mathrm{~A}$ high incidence of medication errors related to IV therapy was found in Germany, where $23 \%$ of the total medication errors occur during IV administration. ${ }^{16,17}$ In Ethiopia, the prevalence of medication administration errors (MAEs) was reported to be $55 \% .^{18}$

MAEs can affect patient morbidity and mortality. They can also influence patients, familiesm, and health care providers indirectly by cost implication, prolonged hospital stays and psychological impact. ${ }^{11}$ Although medication errors are well investigated in many developed countries, research on the issue has rarely been conducted in developing countries, including Ethiopia. Therefore, the aims of this study, which was conducted at Ayder Referral Hospital (ARH), are to determine the prevalence of MAEs and identify the factors associated with them.

\section{Methods and participants}

The study was conducted at ARH, which is located in Mekelle city, Tigray, Ethiopia. The hospital is affiliated to Mekelle University and is the only referral hospital in the region. It has a total capacity of 500 inpatient beds and serves 250-400 cases per day. We conducted an institutional-based cross-sectional study. The sample size needed was determined using single mean population proportion formula. Considering 1.96 for the standard normal variable with $5 \%$ level of significance ( $\alpha$-value), 95\% confidence interval, $5 \%$ margin of error and $10 \%$ contingency for loss, the sample size was calculated to be 384. Because the hospital has 206 beds in the three wards (medical, surgical, and gynecological), the minimum sample size needed was estimated to be 134. Simple random sampling was used to select the study participants. The study included patients aged 18 years and above; patients administered with two or more IV medications during their stay in the ward; and patients admitted to the three wards during the study period. Patients treated with oral or topical medications were excluded from the study.

Data were collected by directly observing medication administrations, and the medical records were reviewed using the pretested data collection format, which was developed by reviewing reputable literatures. All relevant data were collected prospectively from patients' medical records and by direct observation. Demographic information about the patients was obtained from their medical cards and medication administration records. Data on medication administration were collected by directly observing all day-time (6:00 am to $6: 00 \mathrm{pm}$ ) medication administration. Along with the day time observations, medication chart review was carried out to collect data on the off duty (7:00 pm to 6:00 am) drug administration. The observations and the information recorded in the medication charts were documented during the data collection, including all details about the patient's medication regimen. The data were collected by two undergraduate pharmacists employed outside of ARH. Pretest was done on ten patients who were randomly selected from the hospital. These were then excluded from the analysis and amendment was done in the data abstraction format. MAEs were identified by comparing medication administration observed/found as per the order of prescribers. The primary outcome of the study was presence of MAE; age, comorbidity, number of medications per patient, regimen complexity, sex, diagnosis, and dose and frequency of medications were the independent variables studied.

The data were coded, cleaned and checked for completeness. They were entered in Epidata version 3.1 and analyzed using Statistical Package for Social Sciences version 20. Bivariate logistic regression analysis was used to find the association between MAE and independent variables. Variables with $P<0.05$ in the binary logistic regression were reanalyzed using multivariate logistic regression analysis to identify the determinants of MAE. A $P$ value $<0.05$ was considered to be statistically significant. Ethical clearance was obtained from the Institutional Review Board of College of Health Sciences, Mekelle University. Oral consent were obtained from the patients before the data were collected.

\section{Operational definitions}

MAE is a deviation from the physician's medication order as written on the patient's chart. It includes poor medication administration record on the patient chart and failure to comply with the prescription order (drug, dose, dosage regimen, dosage form and length of therapy). ${ }^{19}$

Complex regimen is the prescription of three or more drugs to one patient at the same time. ${ }^{19}$

\section{Results}

\section{Demographic characteristics}

In our study, 134 patients were included, making up a 100\% response rate. More than half of the study participants were males $76(56.7 \%)$ and $58(43.3 \%)$ were in the age group of $40-59$ years. The mean age of the participants was $35 \pm 15.5$ years (Table 1).

\section{IV medication usage information}

More than half of the patients (64.2\%) were admitted to the emergency department before they were transferred to one 
Table I Age and sex distribution of patients $(\mathrm{N}=134)$

\begin{tabular}{ll}
\hline Variables & Frequency (\%) \\
\hline Sex & \\
Male & $76(56.7)$ \\
Female & $58(43.3)$ \\
Age (years) & \\
$18-39$ & $28(20.9)$ \\
$49-59$ & $58(43.3)$ \\
$60-79$ & $33(24.6)$ \\
$80-101$ & $15(11.2)$ \\
\hline
\end{tabular}

Table 2 Characteristics and IV medication usage of patients

\begin{tabular}{ll}
\hline Variables & Frequency (\%) \\
\hline Admission ward & \\
Medical ward & $73(4.5)$ \\
Surgical ward & $35(26.1)$ \\
Gynecology ward & $26(19.4)$ \\
Regimen taken & \\
Simple & $68(50.7)$ \\
Complex & $66(49.3)$ \\
Number of drugs & \\
Two drugs & $67(50.0)$ \\
Three drugs & $43(32.1)$ \\
Four drugs & $17(12.7)$ \\
Five drugs & $7(5.2)$ \\
\hline
\end{tabular}

Abbreviation: IV, intravenous.

of the three wards, and $73(54.5 \%)$ patients were admitted to the medical ward. Concerning the number of drugs per prescription, 67 (50\%), 43 (32.1\%) and $17(12.7 \%)$ patients received two, three and four drugs per prescription, respectively (Table 2 ). An average of 2.73 (2.73 \pm 1.34 : mean \pm standard deviation [SD]) drugs were prescribed at the same time and $37(27.6 \%)$ patients were diagnosed with comorbidities including infection and abscess (Table 3).

\section{MAE}

A total of 366 medication administration interventions had occurred in the studied wards of ARH. Out of the 366 medication administration interventions, 169 (46.1\%) medications administered were labeled as MAE. Among all MAEs, missed dose and wrong dose contributed to 162 (95.8\%) and 7 (4.2\%) errors, respectively. The MAEs were observed in each study site: $89(52.6 \%)$ in the medical ward, $51(30.1 \%)$ in the surgical ward and $29(17.1 \%)$ in the gynecology ward.

\section{Factors associated with IV MAE}

Binary logistic regression analyses showed that age groups 60-79 years (crude odds ratio $[\mathrm{COR}]=1.31,95 \%$ confidence interval $[\mathrm{CI}]=0.47-3.68)$ and $80-101$ years $(\mathrm{COR}=1.13,95 \%$ $\mathrm{CI}=1.02-4.03$ ) were significantly associated with MAEs. Multivariate binary logistic analyses of factors showed that patients
Table 3 Comorbidities

\begin{tabular}{ll}
\hline Comorbidities & Frequency (\%) \\
\hline DM & $5(3.7)$ \\
HIVIAIDS & $4(3)$ \\
TB & $9(6.7)$ \\
Cardiac disease & $11(8.2)$ \\
Hypertension & $2(1.5)$ \\
Cancer & $10(7.5)$ \\
Renal and liver disease & $12(9)$ \\
Infection and abscess & $37(27.6)$ \\
Accident & $5(3.7)$ \\
Others & $39(29.1)$ \\
\hline
\end{tabular}

Abbreviations: AIDS, acquired immunodeficiency syndrome; DM, diabetes mellitus; HIV, human immunodeficiency virus; TB, tuberculosis.

in the age group of 60-79 years (adjusted odds ratio [AOR] $=$ $2.17, \mathrm{CI}=1.532-8.78)$ and $80-101$ years $(\mathrm{AOR}=1.52, \mathrm{CI}=$ 1.198-5.58) were determinants of MAE (Table 4). Patients in the age group of 60-79 years were two times more likely to have MAE than those in the age group of 18-39 years. Moreover, patients in the age group of 80-101 years were 1.5 times more likely to have MAE than those in the age group of 18-39 years.

\section{Discussion}

In this study, the prevalence of MAEs was found to be $46.1 \%$. Among the total medication errors in intervention, missed dose and wrong dose accounted for $95.8 \%$ and $4.2 \%$, respectively. Multivariate binary logistic analyses of factors showed that patients in the age group of $60-79$ years $(\mathrm{AOR}=2.17$, $95 \% \mathrm{CI}=1.53-8.78)$ and $80-101$ years $(\mathrm{AOR}=1.52,95 \%$ $\mathrm{CI}=1.19-5.58)$ were the determinants of MAE.

The rate of MAEs was found to be lower in this study than that conducted at the Jimma University Specialized Hospital (JUSH), Southwest Ethiopia, where 51.8\% MAEs was reported. ${ }^{11}$ The plausible justification for the difference might be the difference in study setting. This study was conducted at the surgical, gynecology and medical wards, whereas the study from JUSH was conducted in the intensive care unit (ICU), where the number of drugs given per patient was more as compared to that administered to patients admitted in the medical wards of ARH because IV administration is highly followed for patients admitted to the ICU in comparison to other wards.

Besides, a much lower rate of MAE was found in our study as compared to that in a prospective observational study conducted at a tertiary care hospital, HospSel, Malaysia, where the rate of MAE was $88.6 \%$. The reason for this difference could be due to the high number (ten or more) of IV drugs prepared and administered at that point of time and the lack of staff nurses (three or less) at the particular ward of the tertiary care hospital. ${ }^{15}$

The rate of MAEs in our study was higher than that in the findings reported from an observational study conducted 
Table 4 Multiple logistic regression analysis of different variables with IV medication administration error in medical, surgical and gynecology wards of ARH from March to April 2015

\begin{tabular}{|c|c|c|c|c|}
\hline \multirow[t]{2}{*}{ Variables } & \multicolumn{2}{|c|}{ Medication administration errors } & \multirow[t]{2}{*}{ COR $(95 \% \mathrm{Cl})$} & \multirow[t]{2}{*}{ AOR $(95 \% \mathrm{Cl})$} \\
\hline & Yes (\%) & No (\%) & & \\
\hline \multicolumn{5}{|c|}{ Age categories (years) } \\
\hline $18-39$ & $12(42.9)$ & $16(57.1)$ & 1 & 1 \\
\hline $40-59$ & $12(20.7)$ & $46(79.3)$ & $2.875(1.077-7.674)$ & $4.501(0.300-15.578)$ \\
\hline $60-79$ & $12(36.4)$ & $21(63.6)$ & $1.312(0.47-3.68 I)$ & $2.166(1.533-8.799)$ \\
\hline $80-101$ & $6(40.0)$ & $9(60.0)$ & $1.125(1.02-4.029)$ & $1.52(1.198-5.584)$ \\
\hline
\end{tabular}

Abbreviations: AOR, adjusted odds ratio; ARH, Ayder Referral Hospital; $\mathrm{Cl}$, confidence interval; COR, crude odds ratio; IV, intravenous.

at three large teaching hospitals in the UK and Germany, which showed 93 (34\%) administration errors from 278 IV interventions observed. ${ }^{19}$ This might be due to the less knowledge, skill and attitude of the health care providers and the difference in provision of health care services to the patients in ARH, as compared to those of UK and German hospitals. The other plausible reason could be due to the involvement of drug supply and pharmacy services in medication administration process in the three large teaching hospitals which resulted in lower frequency of MAE, ${ }^{19}$ as compared to our study in which there was no pharmacist involved in IV administration.

In this study, missed dose was found to be the common type of administration error. The prevalence of missed dose was much higher than that reported in JUSH (18.3\%). This might be due to the difference in study setting. The study from JUSH was conducted at the ICU, wherein patients are kept under close supervision as compared to those in other wards. ${ }^{11}$ Poor availability of medications and poor logistic system of the hospital might also account for the health care professionals missing the medication doses, which could ultimately increase the prevalence of missed dose.

Multivariate binary logistic analyses of factors showed that patients in the age group of $60-79$ years $(\mathrm{AOR}=2.166$, $\mathrm{CI}=1.532-8.799)$ and $80-101$ years $(\mathrm{AOR}=1.52, \mathrm{CI}=$ 1.198-5.584) are determinants of MAEs. Patients in the age group of 60-79 years were two times more likely to have MAEs than patients aged 18-39. Moreover, patients in the age group of $80-101$ years were 1.5 times more likely to have MAEs than patients aged 18-39 years. These findings strengthens the fact that elderly people under chronic medication and with insufficient control of their medication level are one of the risk groups for MAE. ${ }^{20,21}$

\section{Limitations}

This study has some limitations. As this is a cross-sectional study, it might lead to recall bias. Further, the study was conducted at a single center with a small sample size, which might limit the generalization of the findings.

\section{Conclusion}

This study reports a high prevalence of MAEs, of which missed dose was the most common type of error. In addition, it was found that MAEs were influenced by a patient's age. These findings emphasize that health care providers could pay due attention to the risks of MAEs and the factors associated with them.

\section{Acknowledgments}

We would like to acknowledge Mekelle University for cooperating with us to conduct the study. Our deepest gratitude also goes to the staff of Ayder Referral Hospital who helped us in collecting data. The authors received no specific funding for this work.

$\mathrm{TF}$ and EE are assistant lecturers in Mekelle University. MT is a microbiologist in Aksum University. SWA is a clinical pharmacist and lecturer in Mekelle University.

\section{Author contributions}

TF was involved in the conception and design of the study, developed data collection tools and analyzed data. SWA, MT and EE were involved in manuscript writing, analysis and editing. All authors contributed toward data analysis, drafting and revising the paper and agree to be accountable for all aspects of the work.

\section{Disclosure}

The authors report no conflicts of interest in this work.

\section{References}

1. Glavin RJ. Drug errors: consequences, mechanisms, and avoidance. $B r$. J Anaesth. 2010;105(1):76-82.

2. Alsulami Z, Conroy S, Choonara I. Medication errors in the Middle East countries: a systematic review of the literature. Eur J Clin Pharmacol. 2013;69(4):995-1008.

3. Anselmi ML, Peduzzi M, Dos Santos CB. Errors in the administration of intravenous medication in Brazilian hospitals. J Clin Nurs. 2007; 16(10):1840-1841.

4. Cayo L. Compatibility of commonly used IV drugs. Pharm Pract News Spec Ed. 2013;39-46.

5. Zeiter B, Bellers S, Kraemer I. Risk categorization of standardized continuous injection/-infusion solutions at the university medical center Mainz. Eur J Hosp Pharm. 2014;20:58-60.

6. Foinard AM, Simon N, Barthelemy C, Lannoy D, Decaudin B, Odou P. Drug incompatibilities: a problem in clinical practice. J Clin Pharm. 2013;5:34-43. 
7. Kunle R, Godwin AA, Kehinde O, Abraham D. Medication administration error in anaesthetic practice, IOSR-JPBS. 2014;9(6):62-67.

8. Gikic M, Di Paolo ER, Pannatier A, Cotting J. Evaluation of physicochemical incompatibilities during parenteral drug administration in a pediatric intensive care unit. Pharm World Sci. 2000;22(3):88-91.

9. Tissot E, Cornette C, Demoly P, Jacquet M, Barale F, Capellier G. Medication errors at the administration stage in an intensive care unit. Intensive Care Med. 1998;25(4):354-355.

10. Cousins DH, Sabatier B, Begue D, Schmitt C, Hoppe-Tichy T. Medication errors in intravenous drug preparation and administration: a multicenter audit in the UK, Germany and France. Qual Saf Health Care. 2005;14(3):190-195.

11. Agalu A, Ayele Y, Bedada W, Woldie M. Medication administration errors in an intensive care unit in Ethiopia. Int Arch Med. 2012;5(1): $1-6$.

12. McBride-Henry K, Foureur M. Medication administration errors: understanding the issues. Aust J Advanced Nur. 2006;23(3):33-36.

13. Vijayakumar A, Sharon EV, Teena J, Nobil S, Nazeer I. A clinical study on drug-related problems associated with intravenous drug administration. J Basic Clin Pharm. 2014;5(2):49-51.
14. Llewellyn RL, Gordon PC, Reed AR. Drug administration errors-time for national action. S Afr Med J. 2011;101(5):93-98.

15. Ong WM, Subasyini S. Medication errors in intravenous drug preparation and administration. Med J Malaysia. 2013;68(1):52-56.

16. Mansouri A, Ahmadvand A, Hadjibabaie M, Kargar M, Javadi M, Gholami K. Types and severity of medication errors in Iran; a review of the current literature. Daru. 2013;21(1):49-55.

17. Demehin AI, Babalola OO, Erhun WO. Pharmacists and nurses perception of medication errors in a Nigerian University teaching hospital. Int $J$ Health Res. 2008;2(1):51-61.

18. Negash G, Kebede Y, Hawaze S. Medication errors in the adult emergency unit of a tertiary care teaching hospital in Addis Ababa. Arch Pharma Prac. 2013;4(4):8-10.

19. Wirtz V, Taxis K, Barber ND. An observational study of intravenous medication errors in the United Kingdom and in Germany. Pharm World Sci. 2003;25(3):104-111.

20. Zakarov S, Tomson N, Peclova D. medication errors - an ending problem for children and elderly patient. Ups J Med Sci. 2012;117(3):309-307.

21. Szcepura A, Wild D, Nelson S. Medication administration errors for older people in long term residential care. BMC Geriatr. 2011;11:82.
Integrated Pharmacy Research and Practice

\section{Publish your work in this journal}

Integrated Pharmacy Research and Practice is an international, peer-reviewed, open access, online journal, publishing original research, reports, reviews and commentaries on all areas of academic and professional pharmacy practice. This journal aims to represent the academic output of pharmacists and pharmacy practice with particular focus on integrated care. All papers are carefully

\section{Dovepress}

peer reviewed to ensure the highest standards as well as ensuring that we are informing and stimulating pharmaceutical professionals. The manuscript management system is completely online and includes a very quick and fair peer-review system, which is all easy to use. Visit http://www.dovepress.com/ testimonials.php to read real quotes from published authors.

Submit your manuscript here: http://www.dovepress.com/integrated-pharmacy-research-and-practice-journal 